\title{
The Effectiveness of Social Influence Tactics when Used by a Virtual Agent
}

\author{
Gale M. Lucas \\ Institute for Creative \\ Technologies \\ University of Southern California \\ Los Angeles, CA USA \\ lucas@ict.usc.edu
}

\author{
Janina Lehr, \\ Nicole Krämer \& \\ Department of Social Psychology \\ University of Duisburg-Essen \\ Duisburg, Germany \\ nicole.kraemer@uni-due.de
}

\author{
Jonathan Gratch \\ Institute for Creative \\ Technologies \\ University of Southern California \\ Los Angeles, CA USA \\ gratch@ict.usc.edu
}

\begin{abstract}
Research in social science distinguishes between two types of social influence: informational and normative. Informational social influence is driven by the desire to evaluate ambiguous situations correctly, whereas normative social influence is driven by the desire to be liked and gain social acceptance from another person. Although we know from research that humans can effectively use either of these techniques to persuade other humans, scholars have yet to examine the relative effectiveness of informational versus normative social influence when used by virtual agents. We report a study in which users interact with a system that persuades them either using informational or normative social influence. Furthermore, to compare agents to human interlocutors, users are told that the system is either teleoperated by a human (avatar) or fully-automated (agent). Using this design, we are able to compare the effectiveness of virtual agents (vs humans) in employing informational versus normative social influence. Participants interacted with the system, which employed a Wizard-of-Oz operated virtual agent that tried to persuade the user to agree with its rankings on a "survival task." Controlling for initial divergence in rankings between user and the agent, there was a significant main effect such that informational social influence resulted in greater influence than normative influence. However, this was qualified by an interaction that approached significance; users were, if anything, more persuaded by informational influence when they believe the agent was AI (compared to a human), whereas there was no difference between the agent and avatar in the normative influence condition.
\end{abstract}

Permission to make digital or hard copies of all or part of this work for personal or classroom use is granted without fee provided that copies are not made or distributed for profit or commercial advantage and that copies bear this notice and the full citation on the first page. Copyrights for components of this work owned by others than ACM must be honored. Abstracting with credit is permitted. To copy otherwise, or republish, to post on servers or to redistribute to lists, requires prior specific permission and/or a fee. Request permissions from Permissions@acm.org. IVA '19, July 2-5, 2019, PARIS, France

(c) 2019 Association for Computing Machinery.

ACM ISBN 978-1-4503-6672-4/19/07...\$15.00

https://doi.org/10.1145/3308532.3329464

\section{CCS CONCEPTS}

- Human-centered computing $\rightarrow$ Human computer interaction $(\mathrm{HCI}) \rightarrow$ Empirical studies in $\mathrm{HCI}$

\section{KEYWORDS}

Virtual agents, social influence, persuasion, normative influence, informational influence

\section{ACM Reference:}

Gale M. Lucas, Janina Lehr, Nicole Krämer and Jonathan Gratch. 2019. The effectiveness of social influence tactics when used by a virtual agent. In Proceedings of ACM International Conference on Intelligent Virtual Agents (IVA'19). ACM, New York, NY, USA, 8 pages. https://doi.org/10.1145/3308532.3329464.

\section{Introduction}

Humans are prone to social influence; we exhibit a variety of behaviors that demonstrate our susceptibility to social influence including conformity, compliance and obedience to authority [12]. Virtual agents have been found to influence us in similar ways as humans do [3]; for example, virtual agents have been shown to evoke the same kind of established behaviors that result from social influence, such as conformity, compliance, and persuasion [4]. Virtual agents can be used to induce a variety of positive behaviors, including in areas with pertinent long-term consequences such as health, education and pro-environmental behavior (for a review, see [5]). For example, in one study, computer agents provided information and encouragement to individuals with high blood pressure, which improved participants' attitudes and behaviors towards maintaining a healthy blood pressure [6]. Additionally, a good deal of research [7-16] has more directly examined the effectiveness of social influence techniques when used by virtual agents during traditional, well-established persuasion tasks like the lunar survival task [17]. In this prior work, various factors have been shown to impact the effectiveness of virtual agents' attempts at social influence, including use of rapport building techniques, emotional expressions and conversation errors [7-13]. 
However, scholars have yet to examine the relative effectiveness of informational versus normative social influence when used by virtual agents: informational social influence is driven by the desire to evaluate ambiguous situations correctly, whereas normative social influence is driven by the desire to be liked and gain social acceptance from another person. An example of informational influence would be if people are standing at the side of a building looking up at the roof, others passing by conform and also look up; in this case, they do so because the situation is ambiguous (i.e., not a clear, obvious answer) and they are looking to the crowd for cues as to what is going on. In contrast, reciprocity is an example of normative influence (e.g., "if I make the dinner for us, will do the dishes?").

Indeed, while humans can use either of these techniques effectively, computer systems designed to persuade users typically employ primarily informational social influence [7-16]. Yet, it is possible that agents might be able to employ normative social influence, as they have successfully used other relational tactics in interacting with humans such as rapport building [1825], ice-breaking [9-12] and mimicry [26-27]. To explore the ability of agents to use relational tactics in persuasion contexts, we examine how well virtual agents can use normative social influence (compared to information social influence) - and in contrast to how effective these two kinds of social influence are when users believe another human is trying to persuade them.

Accordingly, in this paper we compare the effectiveness of informational and normative social influence when used by a virtual human. However, as a comparison, we also consider how effective informational and normative social influence are when used by a person (thus, ultimately, a $2 \times 2$ design). In sum, while humans often use either normative or information influence on others, and both have been found to be effective when employed by human influencers [1], in this research, we explore the relative effectiveness of these techniques when employed by virtual humans. The remainder of the paper presents background on survival tasks, social influence and agency; describes the experiment; and presents the results and conclusions.

\section{Current Work}

\subsection{Survival Tasks}

Social influence arises across many contexts; here we study it using a 'survival task,' a classic experimental paradigm where participants rank the importance of a number of items (e.g., items that might help one survive a crash on the moon or being stranded in the desert). These are ranked individually and then re-ranked following a discussion with someone else, and the difference in ranking provides an objective measure of social influence. In addition to providing an objective measure, the fact that this task is used so widely allows for comparison across different fields of research. Survival tasks have been used in fields from psychology to computer science, as well as the interface between the two. For example, participants were less persuaded in a lunar survival task when their teammates communicated via teleconference compared with face-to-face interaction [17,28]. Indeed, as fairly context-independent measures of social influence, survival tasks have become popular for studying this topic in virtual human research [7-16].

\subsection{Social Influence Tactics}

Virtual human research on social influence is not limited, however, to only studies using survival tasks. As mentioned earlier, virtual agents have also used social influence techniques to persuade users to engage in behavior change around health [29-34] or energy conservation [35-39]. In these contexts, some social influence techniques have already been shown to be effective when employed by agents (e.g., reciprocity [37-38]). Additionally, some of this research contributes to our understanding of what social influence tactics might be more (or less) effective when used by virtual agents. For example, participants who received more social feedback from agents were more influenced than those who use feedback that involves no social elements [9-11, 35-38]. Furthermore, in addition to adding a social element, some of this work has directly compared different normative influence techniques to each other. For example, reciprocity tactics (e.g., "if I do something for you, will you do something for me in return?") was found to be more effective than foot-in-the-door techniques (i.e., first asking for a small favor, and once they agree, asking for a larger one) [38].

There is other research suggesting that there are some social influence techniques that virtual humans may not be able to use as well as humans can. Work using survival tasks has found that agents cannot use anger to influence users successfully, although humans can [13]. Furthermore, those studies found that agents were unable to use these strategies because they were seen -by default- as low in power, and thus anger was seen as inappropriate [see also [40]). Likewise, it has been found that virtual humans, when serving as personal trainers, also cannot use persuasion techniques that convey negative affect as well as humans can [41].

While some of this research has shown that, compared to entirely non-social attempts at persuasion (i.e., direct requests), social influence tactics that involve more social elements (e.g., reciprocity, banter) are more effective at influencing users [9-11, 35-38], the comparison has not yet been made to informational influence tactics. Distinguishing informational social influence from normative social influence tactics, as described above, is a ubiquitous distinction in the social science literature on influence [1,42]. Sherif famously used the autokinetic effect -an illusion where a small, stationary spot of light projected in a dark room will appear to move- to study informational influence (see [1, 42]). He found that, because the situation is ambiguous (i.e., not a clear, obvious answer due to the illusion) and the participants were thus looking to each other for cues as to the location of the light, participants who were exposed to the autokinetic effect in a group conformed with others' estimates of the position of the light. In contrast, reciprocity and foot-in-the door techniques that we have already discussed are examples of normative influence, and as is the door-in-the-face technique (where one asks for an unreasonable request, and then -once turned down- make a relatively smaller request, which seems 
like nothing in comparison). While humans often use either normative or information influence on others, and both have been found to be effective when employed by human influencers $[1,42]$, in this research, we explore the relative effectiveness of these techniques when employed by virtual humans.

\subsection{Agency}

In Nass and colleagues "media equation," they posit that people will respond fundamentally to media (e.g., fictional characters, cartoon depictions, virtual humans) as they would humans [3]. Further, to the extent to which virtual humans are able to communicate and mimic human behavior, we will perceive and treat them like we treat humans [3]. To test this possibility, they considered whether politeness rules that apply for humanhuman interaction can be transferred to human-computer interaction. Indeed, when asked to rate the performance of an advice-giving agent, users tried to be polite like they would when rating humans [3]. In contrast, people do not afford such consideration to other virtual objects (without human-like features) [43].

In line with the media equation, subsequent work has considered to what extent people treat actors differently based on whether they are real people or artificial intelligence, a distinction which is often referred to as perceived agency [44]. Research usually studies this distinction by comparing agents to avatars. Both agents and avatars are depicted as media, whether it be cartoon depictions or realistic virtual humans; in fact, an agent and an avatar could look identical (and they do in our study). Perceived agency is about who or what users believe is behind the depiction. Avatars are operated by human users, whereas agents are controlled by artificial intelligence of some kind [45]. So, when researchers manipulate perceived agency, they tell users that the virtual human is either controlled by an AI (agent) or a human (avatar) [41, 46-47]. Using this comparison, there is some evidence that the social influence of agents is comparable to that of humans [48-50].

However, users will not always treat agents as if they were humans. There are some contexts where virtual humans are treated differently. Research has found that people respond more positively to agents than avatars in interview settings [46] and in negotiations [47]. Therefore, it is possible that agents and humans might be treated differently in joint decision-making tasks like the survival tasks used here. More importantly, because work in such decision-making contexts has found that agents cannot use the same techniques (like negative affect) to influence users like humans can $[13,41]$, we expected that the virtual human could be more or less effective using informational or normative social influence depending on whether the participants believe that it is an agent or an avatar. We explored this possibility by running the experiment described in the following section.

\section{Experiment}

\subsection{Design}

Participants were randomly assigned to believe that the agent was controlled by a human operator ("Wizard of Oz") or a fully automated system (AI). The participant, therefore, believed that they were either interacting with an avatar or an agent, respectively. They were also exposed to one of two social influence conditions: informational or normative social influence. Accordingly, the purported agent (or avatar) either tried to persuade the participant by providing information about the items in the task, or by providing personal pleas and evoking norms (e.g., the norm of reciprocity), respectively. Thus, the experiment represented a 2 (agency: avatar or agent) $\mathrm{x} 2$ (influence tactic: informational or normative) design. The design was fully between-subjects, so participants were assigned to one of these 4 conditions.

\subsection{Participants}

One hundred and twenty-one participants were recruited from Craigslist for this experiment. They were paid for their participation. Sixty-five participants were female and 56 were male. Participants ages ranged from 18 to 64, with an average of $35.82(\mathrm{SD}=13.10)$.

\subsection{Procedure}

Participants were seated at a table facing a computer screen that displayed our virtual agent, Julie (see Figure 1). The agent used a human-sounding voice generated through text-to-speech to have a conversation with them. During this interaction, they were randomly assigned to believe that the agent was controlled by a human operator (avatar) or a fully automated system (agent). Specifically, the experimenter introduced participants to the virtual agent using graphic representations as well as verbal descriptions that manipulated agency. In the agent condition, participants were led to believe that they were interacting with a computer. They were told: "Our virtual human uses artificial intelligence to have a conversation with you, yet preserves your anonymity. The system gets audio and visual input from you. It uses a Speech recognition tool to understand what you're saying,

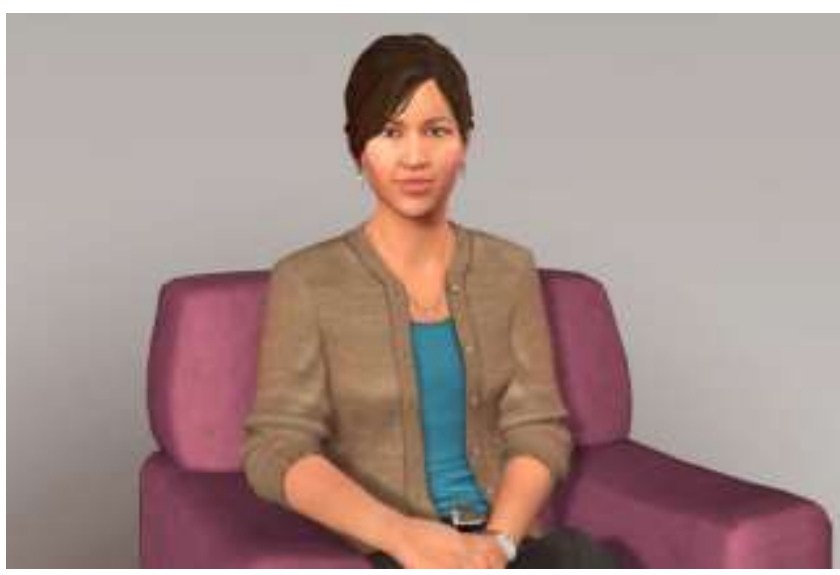

Figure 1: Persuasive agent, Julie 
then uses a complex series of equations to choose the best way to respond." In the avatar condition, participants were led to believe that they were interacting with a person through the virtual human. They were told: "Our virtual human is like a puppet. It allows a person in another room to have a conversation with you, yet preserves your anonymity. My colleague will be sitting in the other room, and be able to see and hear you on this screen. She has access to a set of prerecorded questions and responses that will be used to have a conversation with you."

In reality, in both conditions the study used a Wizard-of-Oz paradigm to determine the behavior of the virtual human. Participants wore a close-talking microphone, which recorded their speech throughout the interaction and transmitted it to the Wizard; and a video camera recorded their face and upper body movements, which was also transmitted to the Wizard. Based on what the Wizard observed, she used a pre-determined script to decide which lines to employ at what time during the survival task.

In both agency conditions, participants completed a joint decision-making task - the Save-the-Art Task [10] - with Julie. The Save-the-Art task is a survival task like the lunar and desert

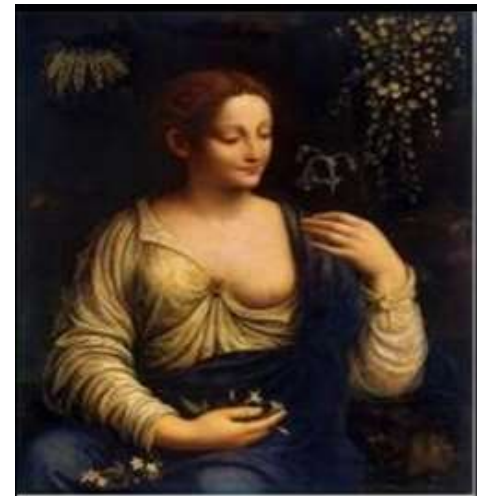

Flora, Francesco Melzi (oil on canvas)

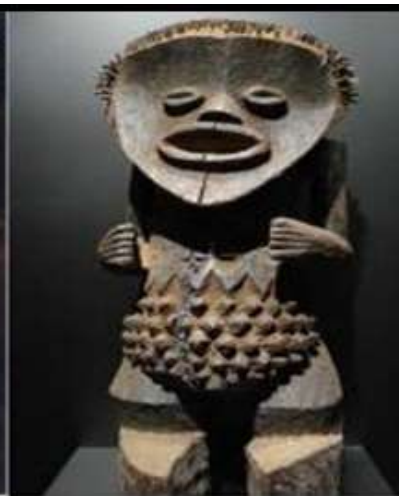

Mambila Figure, Nigeria, unknown (wood)

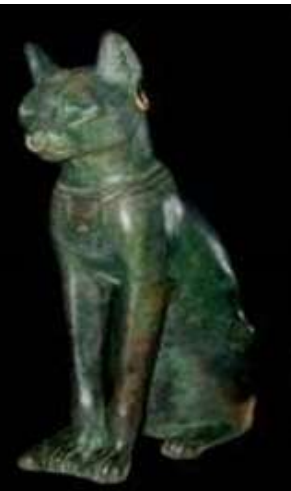

Gayer-Anderson Cat, unknown (bronze statue)

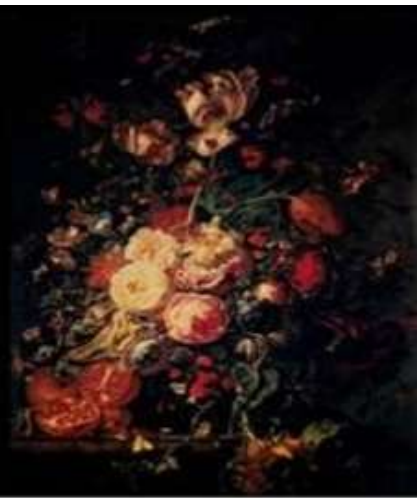

Basket of Flowers by Rachel Ruysch (oil on canvas)
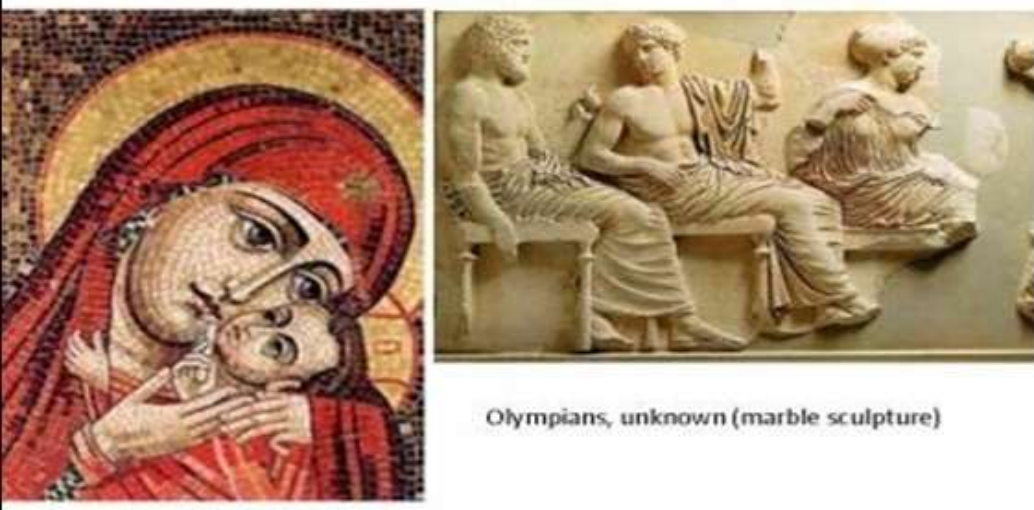

Olympians, unknown (marble sculpture)

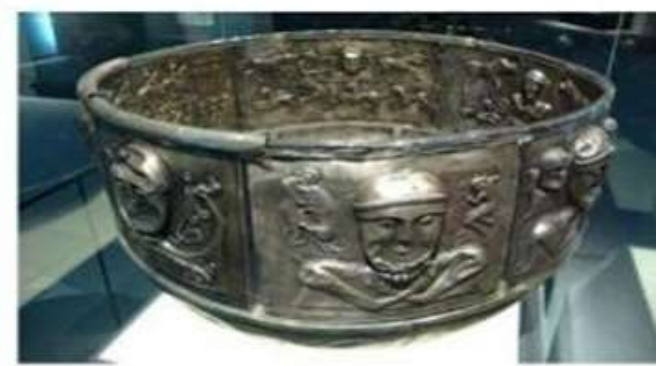

Gundestrup Cauldron, Celtic, unknown (silver, gold, tin, glass)

Madonna \& Child, unknown (ceramic)

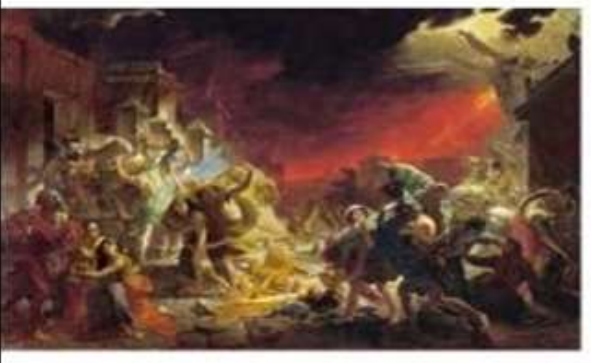

The Last Day of Pompeii, Karl Pavlovich Briullov (oil on canvas)

Figure 2: Items in the Save-the-Art task

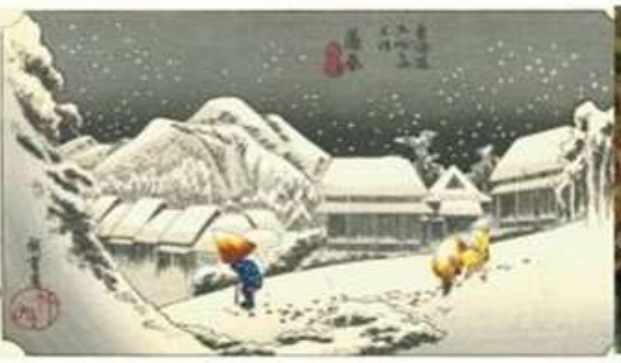

Evening Snow at Kanbara by Utagawa Hiroshige (woodblock print; ink and color on paper).

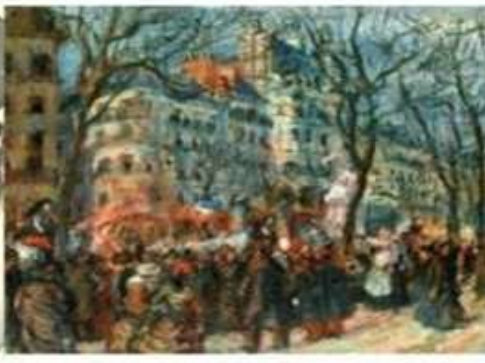

Carnival on the Grand Boulevards, Raoul Dufy (oil on canvas) 
survival tasks described in Section 2.1. For this survival task, participants were asked to imagine that they were a manager at a museum that was on fire. They were asked to rank 10 pieces of art (depicted in Figure 2) as to their importance for being saved. Participants ranked these items individually, and were then told that they should rank them again after discussing it with another member of the emergency management team, Julie (either described as an avatar or agent, depending on agency condition). Participants then engaged in dialogue with Julie about the 10 pieces of art. The participants used a tablet to rank the items initially, and then used the tablet again to re-rank the items (if they wished to do so) after talking to Julie.

During this interaction, Julie used either informational or normative social influence tactics when users did not agree with her. Participants in the informational condition received factual arguments from the agent for ranking the items in that same the agent for ranking the items in a specific order. In contrast, those in the normative condition instead received personal order. For example, in the informational condition, the agent explained that the user should rank the cat statue last because it is made of resilient metal (and therefore least likely to be destroyed in the fire) and it was already damaged anyway. In contrast, the agent did not provide this kind of information in the normative influence condition. Instead, in the normative condition, she explained that the user should rank the statue last because she's had bad experiences with cats, and therefore hates cats and would prefer the cat statue be ranked last.

Similar arguments were used to try to convince participants to rank other art work higher than they had; for example, in the informational condition, the virtual human explained that the user should rank the painting of flowers second because there are few paintings by women artists from that time and, because of the material, it will burn easily. In the normative condition, she instead stated that the user should rank the painting of flowers second because she loves flowers and colorful paintings, especially those from this period painted by women.

In addition to such personal arguments, in the normative condition the virtual human also used other normative techniques: foot-in-the-door, door-in-the-face, and reciprocity [1]. All three of these normative social influence tactics were used in the normative condition; specifically, throughout the dialogue, the virtual human asked several questions to evoke these techniques. She said: 1) Since you didn't rank that last piece based on my suggestion, will you rank this one like I did? [door-in-the-face]; 2) So you changed the ranking of the last piece of art like I suggested, maybe you could also rank another one like I did? [foot-in-the-door]; and 3) If, for the final rankings, I change my ranking of the last item based on your suggestion, would you change your ranking of this one to match mine? [reciprocity]. Agents' rankings were the same in both conditions.

Since participants re-ranked the items after this conversation with Julie, the differences between initial rankings and final rankings served as a measure of influence (as in [10]). The Kendall $\tau$ distance between the participant's and agent's rankings, which ranges from 0 (identical rankings) to 45 (maximally different rankings), was used for both initial rankings and final rankings; influence -the difference between Kendall $\tau$ for initial rankings and Kendall $\tau$ for final rankingstherefore ranges from -45 to 45 , with larger numbers indicating more influence, and zero indicating no influence.

\section{Results}

A 2 (agency: agent or avatar) x 2 (influence tactic: informational or normative) ANCOVA was run on influence -differences between initial rankings and final rankings, as described above. Because initial agreement with Julie limits influence, Kendall $\tau$ for initial rankings was entered as a co-variate. There was a

Influence

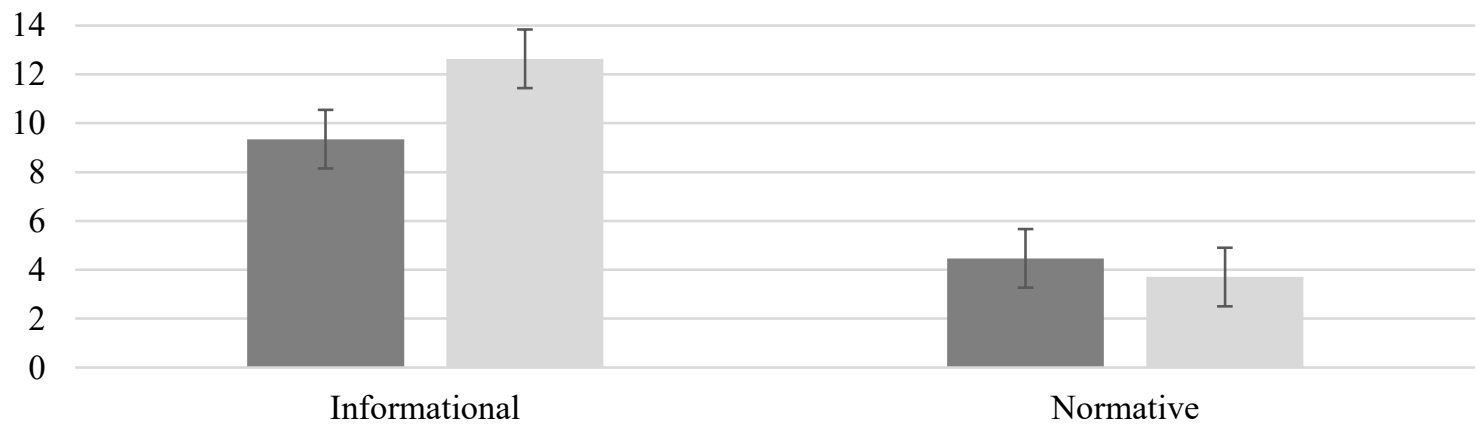

- Human $\quad$ AI

Figure 3: Effect of agency and social influence tactic 
significant main effect of influence tactic $(F(1,116)=34.26, p<$ .001). Controlling for extent of initial agreement with Julie, participants were significantly more influenced in the informational influence condition $(M=11.00, S E=0.85)$ than the normative influence condition $(\mathrm{M}=4.09, \mathrm{SE}=0.82)$. That is, the participants revised their answers more -in order to be closer to Julie's- when she provided information than when she used reciprocity, foot-in-the-door, door-in-the-face and personal appeals.

However, this was qualified by an interaction with agency that approached statistical significance $(F(1,116)=2.96, p=$ .088). As depicted in Figure 3, surprisingly, users were even more persuaded by informational influence when they believe the virtual human was run by a computer (compared to a human; $M$ $=12.64, \mathrm{SE}=1.21$ vs. $\mathrm{M}=9.35, \mathrm{SE}=1.21$ ), whereas there was no such difference between the influence of an agent and avatar in the normative influence condition $(M=3.71, S E=1.16$ vs. $M=$ 4.47, $\mathrm{SE}=1.17)$. The main effect of agency, however, did not approach significance $(F(1,116)=1.10, p=.30)$.

Overall, these results have demonstrated that informational social influence seems to be more effective than normative social influence, at least in the context of a "survival" joint decisionmaking task. Furthermore, if anything, informational influence is even more effective when it is employed by an agent (than an avatar operated by a human).

\section{Discussion}

In this experiment, we found that -when used by a virtual human- informational social influence was more effective than normative social influence. Specifically, in the context of a "survival" joint decision-making task, participants revised their answers more to be in line with arguments made by an agent (or avatar) when it used informational influence than when it used normative social influence. The Save-the-Art task in particular was chosen because -compared to other survival tasks- it is more appropriate for normative social influence; however, because these normative influence tactics were not effective here, it is unlikely that they would be more effective for other such joint decision-making tasks. That is, if participants did not find normative social influence tactics compelling in this context, it seems unlikely that they would be influenced when considering what items are needed to survive a crash landing on the moon or being stranded in the desert (i.e., lunar or desert survival tasks, respectively). While techniques like reciprocity and foot-in-thedoor or door-in-the-face have some chance of being useful in those survival tasks, personal arguments would not (e.g., it is highly unlikely that people would find arguments like "you should rank the oxygen first because I love to go to Oxygen Bars" or "you should rank the water first because I find water tasty" even a little bit compelling when deciding on how to rank these items in the lunar or desert survival tasks).

While we can thus say with relative confidence that our results would generalize to other such joint decision-making tasks, it is possible that the relative weight of informational over normative social influence might be different in other persuasion contexts. For example, normative social influence could still be effective for agents designed to engage in behavior change around health or energy conservation (as in [29-39]). Indeed, some work suggests that it might. Participants who received more social arguments from agents are more likely to conserve energy than those who use arguments that involve no social elements [35-38]. Therefore, in such contexts, normative social influence could be relatively more effective than it was here (specifically, during a "survival" decision-making task).

However, this may not be the case even in these other persuasion contexts. While that prior work did show that agents that used normative social influence tactics were more effective than those that used direct requests without any social dialog, that research did not pit normative social influence tactics specifically against informational influence. When our work is considered with respect to this previous research, it seems that normative social influence tactics (e.g., reciprocity, personal arguments, etc.), might be more effective than direct requests without any social dialog, but they might not be as effective as informational social influence techniques. Future research should consider this comparison.

More generally, it is possible that, in the current research, informational influence was found to be more effective than normative influence because the answers in this task were ambiguous. Because informational influence is driven by the desire to evaluate ambiguous situations correctly, when answers are ambiguous, informational influence might indeed be most effective. If the answers were more obvious (as in the Asch line task [51]), the pressure to "get along" may end up driving behavior more than desire to align one's answers with others in order to be correct. Indeed, if the answers were clear, informational influence could be perceived as superfluous or -if it contradicted what seemed obvious- just flat our wrong. While this may be the case, the kinds of applications that would use persuasive agents are more likely to involve situations where the answer is ambiguous rather than clear-cut. Agents could help affect behavior if users, for example, are trying to figure out which way to go, and would probably not come in to play if users already knew the answer. Accordingly, it is important that we tested these types of influence in an ambiguous situation rather than an unambiguous one. Moreover, just because the situation was ambiguous does not mean that normative influence could not have any impact; indeed, research on such normative influence tactics have included ambiguous situations (see $[1,42]$ ), and found such techniques to be effective in these cases.

Furthermore, this research considered a select set of normative social influence tactics: reciprocity, foot-in-the-door, door-in-the-face, and personal arguments. While this set is broadly representative of normative social influence tactics, it is possible that other such techniques -like "low-balling"- that were not included in this research might be particularly effective, at least in certain persuasion contexts (e.g., selling a car). Indeed, research on negotiations has shown that, like people, intelligent virtual agents can effectively lie about their preferences to influence their opponent and "win" in a negotiation [52-53]. 
Accordingly, other influence techniques not tested here should be considered in future research, as they may be more effective, especially in specific contexts such as negotiation or behavior change. Additionally, the particular way that the normative social influence techniques were implemented in this research could have impacted the effectiveness of these tactics. Along these lines, it is also possible that the current implementation did not completely separate normative from informational influence. Although the informational condition was clearly more informational than normative, participants could have misinterpreted certain lines as conveying some additional normative reasons for changing their rankings. For example, while the phrase "a rare example of a work by a woman in this period" was intended to denote that -because of its rarity- the artwork held a high monetary value, participants could have read into the statement that feminist values were also being asserted. As such, future research could consider alternative implementations and operationalizations for these two types of influence tactics.

Furthermore, this research also found that, if anything, informational influence is even more effective when it is employed by an agent (compared to an avatar operated by a human). These results suggest that intelligent virtual agents in particular can benefit from using informational influence. As discussed elsewhere [40], because agents are often perceived as low in power (see also [13]), this finding may indicate that there is an additional advantage of using informational social influence if the speaker has relatively low power (such as an agent). Accordingly, further research should explicitly manipulate gender (as in [13]) to discern if there is a moderating effect on how useful informational versus normative social influence is for virtual agents. Along these lines, future work could also manipulate the gender of the agent; for example, perhaps male agents -with greater power in some leadership contexts- might be able to use normative social influence more effectively in those contexts.

When designing persuasive technologies that employ social dialogue, designers might also want to consider this finding. Indeed, if the artificial intelligence brings informational influence to bear to the task, it is likely to be more persuasive than if it tries to employ more normative social influence techniques. Our research implies this benefit of informational social influence might be even more potent when the users are focusing on the fact that the agent is artificially intelligent (compared to, say, when they might think crowdsourced human intelligence is being used to drive the system). Therefore, designers may want to emphasize informational social influence (over normative), and might also consider highlighting to users that the technology is driven by an artificial intelligence.

While we found that normative influence, when used on its own, is not as effective as informational influence, it is possible that it could be useful in augmenting informational influence. Instead of using just one or the other, future work could evaluate the benefit of adding normative influence tactics (i.e., reciprocity, foot-in-the-door, door-in-the-face, personal arguments) to informational social influence.
Given the apparent value of informational social influence, future research could try to identify when normative social influence might be useful. Research could, for example, explicitly test the extent to which context or task-type matters for the relative effectiveness of normative versus informational influence. Moreover, normative social influence may be more effective in established relationships than new ones (which we only considered here). Indeed, personal appeals (e.g., "please do this for me") might be more convincing when employed in the context of a longer-term relationship. However, other research suggests that these kinds of appeals can be useful even after just a few exchanges: after less than ten rounds of an iterative social dilemma, agents can more effectively use this kind of relational tactic (i.e., explicitly asking for favors) to influence their human interaction partners [54-56]. To provide clarity around this issue, future research should consider length and strength of relationship with the agent as a possible moderating factor.

It is possible that, like we observed here, informational influence is still more effective than normative social influence; however, it would still be important to identify the boundary conditions or moderators of this effect. From the results of this initial research attempt, though, we would conclude that interlocutors -especially artificially intelligent agents- could benefit more from using informational influence than normative social influence.

\section{ACKNOWLEDGMENTS}

This project received funding from the German Academic Exchange Service through the PROMOS program. The work was also supported in part by the U.S. Army. Any opinion, content or information presented does not necessarily reflect the position or the policy of the United States Government, and no official endorsement should be inferred.

\section{REFERENCES}

[1] Cialdini, R. B., \& Goldstein, N. J. Social influence: Compliance and conformity. Annual Review of Psychology., 55, 591-621. (2004).

[2] Prislin, J., \& Wood, W. Social influences in attitudes and attitude change. In Albarracín et al., Handbook of attitudes, pp. 671-706. (2005).

[3] Reeves, B., \& Nass, C. How people treat computers, television, and new media like real people and places (p. 119). CSLI Publications and Cambridge University press. (1996).

[4] Appel, J., von der Pütten, A., Krämer, N. C., \& Gratch, J. Does humanity matter? Analyzing the importance of social cues and perceived agency of a computer system for the emergence of social reactions during humancomputer interaction. Advances in Human-Computer Interaction, 13, 1-10. (2012).

[5] Fox, J., Christy, K. R., \& Vang, M. H. The experience of presence in persuasive virtual environments. In G. Riva, J. Waterworth, \& D. Murray (Eds.) Interacting with presence: $\mathrm{HCI}$ and the sense of presence in computermediated environments (pp. 164-178). Berlin, Germany: DeGruyter Open. (2014).

[6] Skalski, P., \& Tamborini, R. The role of social presence in interactive agentbased persuasion. Media psychology, 10, 385-413. (2007).

[7] Wang, Y., Khooshabeh, P. \& Gratch, J. Looking real and making mistakes. in International Workshop on Intelligent Virtual Agents, 339-348, (Springer, 2013).

[8] Blascovich, J. \& McCall, C. Social Influence in Virtual Environments. in The Oxford handbook of media psychology, 305-315 (Oxford University Press, 2013).

[9] Lucas, G. M., Boberg, J., Artstein, R., Traum, D., Gratch, J., Gainer, A., Johnson, E., Leuski, A., \& Nakano, M. Getting to know each other: The role of social dialogue in recovery from errors in social robots. Proceedings of the Conference on Human-Robot Interaction, 344-351 (2018). 
[10] Artstein, R., Traum, D., Boberg, J., Gainer, A., Gratch, J., Johnson, E., Leuski, A., \& Nakano, M. Listen to My Body: Does Making Friends Help Influence People? Proceedings of the Thirtieth International Florida Artificial Intelligence Research Society Conference, 430-435 (2017).

[11] Lucas, G. M., Boberg, J., Traum, D., Artstein, R., Gratch, J., Gainer, A., Johnson, E., Leuski, \& M. Nakano. 2018. Culture, Errors, and Rapport-building Dialogue in Social Agents. In IVA '18: 2018 ACM International Conference on Intelligent Virtual Agents, November 5-8 (2018).

[12] Artstein, R., Boberg, J., Gainer, A., Gratch, J., Johnson, E., Leuski, A., Lucas, G. M., \& Traum, D. (2018). The Niki and Julie Corpus: collaborative multimodal dialogues between humans, robots, and virtual agents. In Proceedings of the Eleventh International Conference on Language Resources and Evaluation, 2928-2932 (2018).

[13] Wang, Y., Lucas, G., Khooshabeh, P., de Melo, C., \& Gratch, J. Effects of emotional expressions on persuasion. Social Influence 10(4):236-249. (2015).

[14] Adalgeirsson, S. O., \& Breazeal, C. Mebot: A robotic platform for socially embodied presence. In Proceedings of International Conference on HumanRobot Interaction, 15-22. IEEE Press. (2010).

[15] Takayama, L., Groom, V., \& Nass, C. I'm sorry, Dave: I'm afraid I won't do that: Social aspects of human-agent conflict. In Proceedings of SIGCHI, 20992108. ACM. (2009).

[16] Khooshabeh, P., McCall, C., Gandhe, S., Gratch, J., \& Blascovich, J. Does it matter if a computer jokes? In CHI'11 Extended Abstracts on Human Factors in Computing Systems, 77-86. ACM. (2011).

[17] Barefoot, J., Wiggins, B., \& Latane, B. Computer-Mediated Communication: Decision-making and Informal Interaction. Report for Defense Technial Information Center. (1985).

[18] Bickmore, T., \& Cassell, J. Relational agents: a model and implementation of building user trust. In Proceedings of the SIGCHI conference on Human factors in computing systems, 396-403 (2001).

[19] Bickmore, T. W., Caruso, L., \& Clough-Gorr, K. Acceptance and usability of a relational agent interface by urban older adults. In $\mathrm{CHI} 05$ extended abstracts on Human factors in computing systems, 1212-1215 (2005).

[20] Bickmore, T., \& Gruber, A. Relational agents in clinical psychiatry. Harvard review of psychiatry, 18(2), 119-130 (2010).

[21] Gratch, J., Wang, N., Gerten, J., Fast, E., \& Duffy, R. Creating rapport with virtual agents. In International workshop on intelligent virtual agents, 125-138 (2007).

[22] Gratch, J., Kang, S. H., and Wang, N. Using social agents to explore theories of rapport and emotional resonance. In J. Gratch and S. Marsella (Eds.) Emotion in Nature and Artifact, 181-197, (2013).

[23] Appel, J., von der Pütten, A., Krämer, N. C., \& Gratch, J. Does humanity matter? Analyzing the importance of social cues and perceived agency of a computer system for the emergence of social reactions during humancomputer interaction. Advances in Human-Computer Interaction. 13 (2012).

[24] Huang, L., Morency, L. P., \& Gratch, J. (2011, September). Virtual Rapport 2.0. In International Workshop on Intelligent Virtual Agents (pp. 68-79). Springer, Berlin, Heidelberg.

[25] Schroder, M., Bevacqua, E., Cowie, R., Eyben, F., Gunes, H., Heylen, D., ... \& Pelachaud, C. Building autonomous sensitive artificial listeners. IEEE transactions on affective computing, 3(2), 165-183 (2012).

[26] Bailenson, J. N., \& Yee, N. Digital chameleons: Automatic assimilation of nonverbal gestures in immersive virtual environments. Psychological science $16,10,814-819$ (2005)

[27] Hoegen, R., van der Schalk, J., Lucas, G., \& Gratch, J. (2018, November). The impact of agent facial mimicry on social behavior in a prisoner's dilemma. In Proceedings of the 18th International Conference on Intelligent Virtual Agents (pp. 275-280). ACM.

[28] Werkhoven, P. J., Schraagen, J. M., \& Punte, P. A. J. Seeing is believing: Communication performance under isotropic tele-conferencing conditions. Displays 22(4):137-149. (2001)

[29] Manuvinakurike, R., Velicer, W. F. \& Bickmore, T. W. Automated indexing of Internet stories for health behavior change: weight loss attitude pilot study. $f$ Med. Internet Res., 16, e285 (2014)

[30] Albaina, I. M., Visser, T., van der Mast, C. A., \& Vastenburg, M. H. Flowie: A persuasive virtual coach to motivate elderly individuals to walk. In G. Troester, K. Connelly, \& B. Arnrich (Eds.), Proceedings of the 3d International ICST Conference on Pervasive Computing Technologies for Healthcare. ICST. (2009).

[31] Fox, J., \& Bailenson, J. N. Virtual Self-Modeling: The Effects of Vicarious Reinforcement and Identification on Exercise Behaviors. Media Psychology, 12(1), 1-25. (2009).

[32] IJsselsteijn, W. A., Kort, Y. de, Bonants, R., Jager, M. de, \& Westerink, J. Virtual Cycling: Effects of immersion and a virtual coach on motivation and presence in a home fitness application. In Proceedings Virtual Reality Design and Evaluation Workshop, 22-29 (2004).

[33] King, A. C., Bickmore, T. W., Campero, M. I., Pruitt, L. A., \& Yin, J. L. Employing virtual advisors in preventive care for underserved communities:
Results from the COMPASS study. Journal of health communication, 18(12), 1449-1464 (2013).

[34] Buttussi, F., Chittaro, L., \& Nadalutti, D. Bringing mobile guides and fitness activities together: a solution based on an embodied virtual trainer. In Proceedings of the 8 th conference on Human-computer interaction with mobile devices and services, 29-36 (ACM, 2006).

[35] Ham, J. \& Midden, C. J. H. A persuasive robot to stimulate energy conservation: the influence of positive and negative social feedback and task similarity on energy-consumption behavior. Int. J. Soc. Robot., 6, 163-171 (2014).

[36] Khashe, S., Lucas, G. M., Becerik-Gerber, B., \& Gratch, J. Buildings with persona: Towards effective building-occupant communication. Computers in Human Behavior, 75, 607-618 (2017).

[37] Khashe, S., Lucas, G., Becerik-Gerber, B., \& Gratch, J. Establishing Social Dialog between Buildings and Their Users. International Journal of HumanComputer Interaction, 1-12. (2018).

[38] Khashe, S., Heydarian, A., Becerik-Gerber, B., \& Wood, W. Exploring the effectiveness of social messages on promoting energy conservation behavior in buildings. Building and Environment, 102, 83-94. (2016).

[39] Khashe, S., Becerik-Gerber, B., Lucas, G., \& Gratch, J. Persuasive Effects of Immersion in Virtual Environments for Measuring Pro-Environmenta Behaviors. 34th International Symposium on Automation and Robotics in Construction (2018).

[40] Khooshabeh, P., \& Lucas, G. M. Virtual human role players for studying social factors in organizational decision making. Frontiers in Psychology, 9:194. (2018).

[41] Lucas, G. M., Krämer, N., Peters, C., Taesch, L. S., Mell, J., \& Gratch, J. Effects of Perceived Agency and Message Tone in Responding to a Virtual Personal Trainer. In Proceedings of the 18th International Conference on Intelligent Virtual Agents (pp. 247-254). ACM. . (2018).

[42] Cialdini, R. B. Influence: Science and practice (Vol. 4). Boston, MA: Pearson education. (2009)

[43] Brave, S., \& Nass, C. Emotion in human-computer interaction. HumanComputer Interaction, 53-68. (2003).

[44] Nowak, K. L., \& Biocca, F. The effect of the agency and anthropomorphism on users' sense of telepresence, copresence, and social presence in virtual environments. In Presence. Teleoperators and Virtual Environments, 481-494. (2003).

[45] Bailenson, J. N., \& Blascovich, J. Avatars. Encyclopedia of Human-Computer Interaction. Berkshire Publishing Group. (2004)

[46] Lucas, G. M., Gratch, J., King, A., and Morency, L. P. It's only a computer: Virtual humans increase willingness to disclose. Computers in Human Behavior. 37, 94-100. (2014).

[47] Gratch, J., DeVault, D., \& Lucas, G. M. (2016). The benefits of virtual humans for teaching negotiation. Proceedings of the 16th International Conference on Intelligent Virtual Agent, 276-287.

[48] Baylor, A. L. Pedagogical agents as a social interface. Educational Technology, (47(1)), 11-14. (2007).

[49] Kim, Y., \& Baylor, A. L. Pedagogical agents as social models to influence learner attitudes. Educational Technology, (47(1)), 23-28. (2007).

[50] Lee, J. E., Nass, C., Brave, S., Morishima, Y., Nakajima, H., \& Yamada, R. The Case for Caring Colearners: The Effects of a Computer-Mediated Colearner Agent on Trust and Learning. Journal of Communication, 57(2), 183-204. (2007).

[51] Asch, S. E., \& Guetzkow, H. Effects of group pressure upon the modification and distortion of judgments. Groups, leadership, and men, 222-236. (1951).

[52] Nazari, Z., Lucas, G., \& Gratch, J. Fixed-pie Lie in Action. In International Conference on Intelligent Virtual Agents, 287-300. ACM. (2017).

[53] Mell, J., Gratch, J., Baarslag, T., Aydogran, R., \& Jonker, C. M. Results of the First Annual Human-Agent League of the Automated Negotiating Agents Competition. In Proceedings of the 18th International Conference on Intelligent Virtual Agents, 23-28. ACM. (2018).

[54] Mell, J., Lucas, G. M. \& Gratch, J. An effective conversation tactic for creating value over repeated negotiations. Proceedings of the 14th conference on Autonomous Agents and Multi-Agent Systems, 1567-1576. (2015).

[55] Mell, J., Lucas, G. M., Gratch, J., \& Rosenfeld, A. Saying YES! The crosscultural complexities of favors, fairness, and trust in negotiation. Proceedings of the 6th International Conference on Affective Computing and Intelligent Interaction, 194-200. (2015).

[56] Mell, J., Lucas, G. M., Mozgai, S., Boberg, J., Artstein, R. \& Gratch, J. Towards a repeated negotiating agent that treats people individually: Cooperation, social value orientation, \& machiavellianism. Proceedings of the 18th International Conference on Intelligent Virtual Agents, 125-132. (2018). 